\title{
Ion Core Structures in Anthracene Anion Tetramers
}

\author{
Jeong Hyun Kim. Nam Ki Lee. Sang Hak Lee. Sang Yun Han. ${ }^{a}$ and Jae Kyu Song ${ }^{\dagger,{ }^{*}}$ \\ School of Chemistry, Seoul National University, Seoul 151-747, Korea \\ "Department of Chemistry, Kyninghee University, Seoul 130-701, Korea. "E-mail: jaeksong@khtiac.kr \\ Received May 31, 2006
}

Key Words : Anthracene, Anion, Cluster, Ion core, Density functional theory

Anthracene is a typical example of organic molecular crystals for the study of charge carrier states. ${ }^{1-3}$ The weakness of van der Waals intermolecular interaction makes the molecular properties dominant over the crystalline ones, which leads to localized charge states in molecular crystals. The localized charge carrier polarizes the subsystems of the surrounding lattice, where a polaron-like particle emerges due to many-electron interactions in the crystals. As it provides a unique environment to investigate the physics of polaron, the organic molecular crystal has been a subject of extensive studies. In particular, the study of anthracene anion clusters, as a microscopic model of charge localization, will contribute to understand the nature of polaron in organic molecular crystals.

Despite numerous studies for the core structures of aromatic cation and neutral clusters, little has been investigated for those of anion clusters. ${ }^{4-16}$ Recently, the multiple ion cores and switching of ion cores in the anthracene anion clusters, $\mathrm{An}_{n}{ }^{-}$( $n=1-16$ ), were investigated by anion photoelectron spectroscopy and theoretical calculations. ${ }^{17.18}$ The study revealed that a few different ion cores exist depending on the cluster size. The character of ion core changes from monomeric to dimeric to trimeric, as the size increases from $n=1$ to 3 . For $n=4$, coexistence of two ion cores was proposed to account for the anomalous shape of the photoelectron spectrum, which were tentatively assigned as dimeric and trimeric ion cores. Due to lack of further information, the explanation could be mainly deduced from the optimized geometries of neutral clusters. The ion core becomes monomeric again at $n=5$ with the completion of half-filled solvation shell. It remains still unclear what ion cores represent the unexpected photoelectron spectrum at $n$ $=4$. In this Note, we revisit the ion core structures at $n=4$, and report a few ion core structures found in the stable geometries at $n=4$.

Figure 1 shows the photoelectron spectra of $\mathrm{An}_{n}^{-}$( $n=1-$ 5 ), which are fitted with Gaussian profiles representing the vibration progression of the $v_{6}$ mode of An molecule. The vertical detachment energies (VDEs) obtained from the fitting are presented as solid bars. As previously reported, ${ }^{17-19}$ the spectral shape of $\mathrm{An}_{4}{ }^{-}$is distinctly different from those of the others. The anomalous shape is deconvoluted into two separate vibrational progressions with different

Present address: Nanobio Fusion Research Center, Korea Reseanch Institute of Standards and Science, Daejeon 305-340, Korea

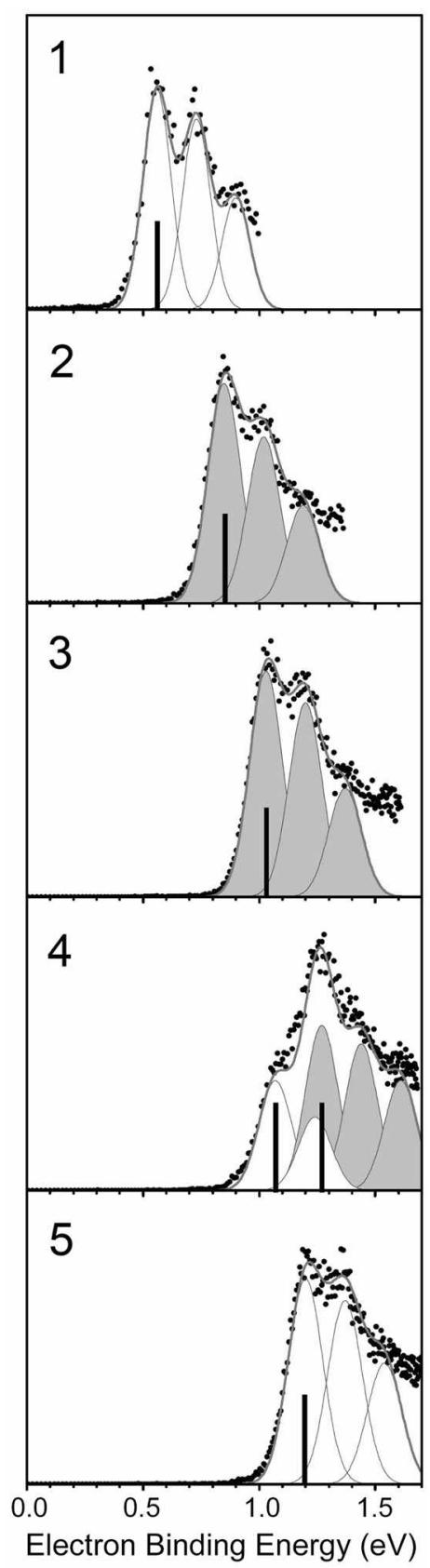

Figure 1. Photoelectron spectra of anthracene cluster anions, $\mathrm{An}_{r}{ }^{-}$ $(n=1-5)$, measured by photodetachment at $532 \mathrm{~nm}$. The spectra are fitted by a set of Gaussian functions with the $v_{6}$ mode separation $\left(1410 \mathrm{~cm}^{-1}\right)$. The solid bars denote their vertical detachunent energies (VDEs). 
(a)

Top view

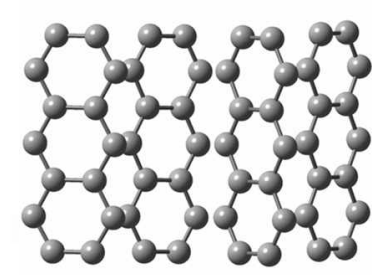

(b)

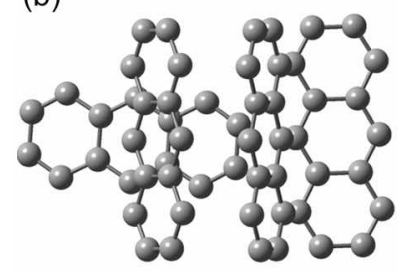

(c)

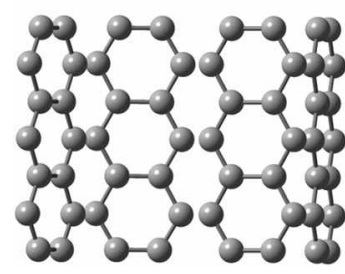

Side view
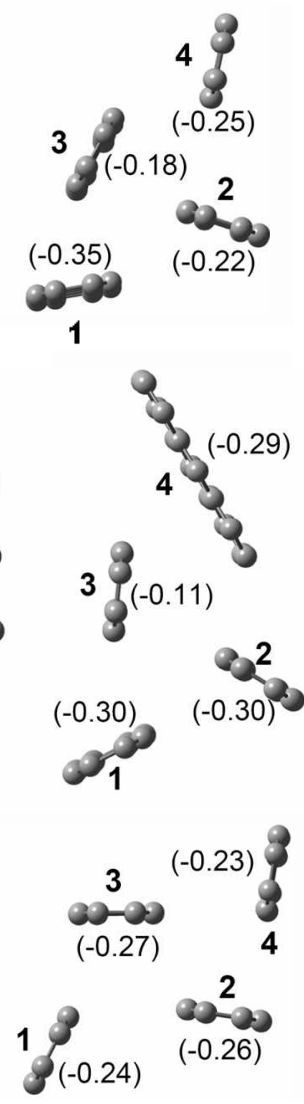

Figure 2. (a) The most stable geometry of the anthracene anion tetramer, $\mathrm{An}_{4}^{-}$. The left column shows a top view, while the right column is a side view. Hydrogen atons are onitted for clarity. The numbers in parentheses represent the charge populations on each constituent obtained by Mulliken population analysis. (b) Another optimized geometry of $\mathrm{An}_{4}{ }^{-}$higher than (a) by $0.03 \mathrm{eV}$. (c) Another syinmetric gconetry of $\mathrm{An}_{4}{ }^{-}$higher than (a) by $0.05 \mathrm{eV}$.

origins, suggesting the coexistence of two ion cores.

In order to address the ion core structures in $\mathrm{An}_{4}{ }^{-}$more carefully, the theoretical calculations were carried out at the density functional theory (B3LYP) using the GAUSSIAN 98 program suite.$^{20}$ Because $\mathrm{An}_{4}{ }^{-}$is a fairly large system, the B3LYP/6-31G leve] without diffusion and polarization functions was employed in order to keep the computational expense affordable. The stable geometries of $\mathrm{An}_{4}{ }^{-}$were calculated using analytical gradient methods with full geometry optimization from several known geometries of the corresponding neutral clusters. ${ }^{16}$ Because the systems of interest have plenty of degrees of freedom with many local minimum states, the optimized geometries were sometimes found to vary with calculation levels. ${ }^{14-16}$

The most stable geometry of $\mathrm{An}_{4}{ }^{-}$is rather asymmetric, which is presented in Figure $2 \mathrm{a}$. With the geometry alone, the most stable geometry seems to consist of the trimeric ion core and one neutral solvent attached to it, since the arrangement of the three moieties, denoted as $1,2,3$ or 2,3 , 4 in Figure 2a, resembles one of optimized geometries of $\mathrm{An}_{3}{ }^{-}$with a trimeric ion core. ${ }^{17}$ However, the charge distri- bution is opposite to what is expected in such an interpretation. The excess electron is more populated in one moiety (1) than in the other three, where the rest of charge is distributed.

In general, $\pi$-hydrogen bonding-type interaction and stacking interaction between aromatic rings are competing factors in detenmining the geometry of neutral aromatic clusters. In neutral polycyclic aromatic hydrocarbon dimers, stacking interaction is more important than $\pi$-hydrogen interaction. Thus, dimers prefer to have a parallel-displaced geometry rather than a T-shaped one. ${ }^{10-16}$ An excess electron in anion dimers can alter the significance of the two factors. In fact, a T-shaped geometry was found in the naphthalenebenzene anion dimer, where the hydrogen atoms of benzene interact with naphthalene anion through $\pi$-hydrogen bonding. ${ }^{21}$ Naphthalene anion dimer was also suggested to have a similar T-shaped geometry. ${ }^{22}$

Delocalization of the excess electron over the An molecule, however, reduces the charge-induced effects. ${ }^{23}$ A parallel-displaced geometry is favored in $\mathrm{An}_{2}^{-}$, which is similar to the optimized geometry of neutral dimer, $\mathrm{An}_{2}{ }^{11,16}$ The possible charge resonance and enhanced stacking interaction due to large molecule size of An contribute to stabilize a parallel-displaced geometry in $\mathrm{An}_{2}{ }^{-17,24,25}$ For Ani, a double parallel-displaced geometry is the most favorable one, where three molecules are not perfectly parallel and the center moiety is a little tilted toward the end moieties, in order to stabilize further by $\pi$-hydrogen bonding. Another stable geometry of $\mathrm{An}_{3}{ }^{-1}$ is a triangular one, ${ }^{17}$ which is quite similar to that of neutral $\mathrm{An}_{3 .}{ }^{1+-16}$ The effect of charge-injection is much weakened by delocalization of the charge over three moieties, which allows the optimized neutral structures still to be valid in anion clusters. In the case of $\mathrm{Ant}_{4}{ }^{-}$, the excess electron can be even more delocalized. The most stable geometry of $\mathrm{An}_{4}{ }^{-}$in Figure $2 \mathrm{a}$ shows charge density distributed over all cluster constituents. The geometry of three moieties $(1,2,3$ or $2,3,4)$ is close to that of neutral trimer clusters, $\mathrm{An}_{3}{ }^{14-16}$

Another stable geometry of $\mathrm{An}_{4}^{-}$appears to be more asymmetric, as shown in Figure $2 b$, which is higher in energy by $0.03 \mathrm{eV}$ than that in Figure $2 \mathrm{a}$. Most of excess electron is evenly populated in three mojeties $(1,2,4)$ and only little is occupied in the other (3). Although the charge population analysis may suggest the trimeric ion core structure, we note that the geometry of trimeric ion core in $\mathrm{An}_{3}{ }^{-}$is totally different from the arrangement of more populated moieties $(1,2,4){ }^{17}$ Figure $2 \mathrm{c}$ presents another stable geometry of $\mathrm{An}_{4}^{-}$, which is more symmetric and higher in energy by $0.05 \mathrm{eV}$ than the one in Figure $2 \mathrm{a}$. The nearly parallel geometry of two moieties $(2,3)$ seems to indicate a dimeric jon core with two neutral solvents $(\mathbf{1}, \mathbf{4})$. However, the excess electron is distributed over the four molecules almost equally, suggesting that ion core structure is more like tetrameric. Interestingly, the geometry is quite similar to the known stable geometry of neutral $\mathrm{An}_{4} .^{1+-16}$ As discussed above, the delocalization of an excess electron reduces the charge-induced effects. However, the presence of the excess 
electron does change the intermolecular distances in the anion clusters, in spite of the seemingly similar geometries between $\mathrm{An}_{4}{ }^{-}$and $\mathrm{An} 4$. As a consequence, the VDE of $\mathrm{An}_{4}{ }^{-}$ with a tetrameric ion core structure can increase, since the vertical transition in photodetachment involves a lot of exited states of intemolecular vibrational modes in the corresponding neutral cluster.

For $\mathrm{An}_{2}{ }^{-}$and $\mathrm{An}_{3}{ }^{-}$, stable geometries are often found to be nearly symmetric. However, as shown in Figure 2, a particular pair of molecules in $\mathrm{An}_{4}^{-}$(1 and 4) is always more separated than other combinations. As the cluster size becomes larger, it is less probable for its constituents to have identical amount of intermolecular interactions. In other words, some molecules may not be affected by the excess electron or ion core as much as their neighbors due to asymmetry of the geometry. Therefore, an excess electron can be more or less localized even in the most stable geometry, as shown in Figure $2 \mathrm{a}$.

In summary, we report the ion core structures in $\mathrm{An}_{4}^{-}$, which supports the coexistence of the ion core structures of different charge-sharing characters in $\mathrm{Arl}_{4}{ }^{-}$. The coexistence and the predicted changes in intermolecular distances may account for the anomalous photoelectron spectrum in $\mathrm{An}_{4}^{-}$.

\section{Experimental Section}

The details of the experimental apparatus have been described elsewhere. ${ }^{18.26}$ The anion clusters was generated by supersonic expansion of anthracene vapor at $170^{\circ} \mathrm{C}$ seeded in Ar carrier gas and by electron attachment to neutral clusters. The anion clusters passed through a $1.8 \mathrm{~m}$ long Wiley-McLaren-type time-of-flight mass spectrometer and were mass-selected. Photoelectron spectra of $\mathrm{An}_{\mu}^{-}$were obtained from photodetachment by using unfocused laser light at $532 \mathrm{~nm}$.

\section{References}

I. Silinsh, E. A. Organic Molecular Cnstals; Springer-Verlag: Berlin Heldelberg New York, 1980.

2. Silinsh, E. A.; Capek, V. Organic Molecular Coystals; AIP press: New York, 1994.

3. Silinsh, E. A.; Klimkâns, A.; Larsson, S.; Capek, V. Chent. Phys. 1995, 198,311 .

4. Badger, B.; Brocklehurst. B. Nature 1968. 219. 263.

5. Neusser, H. J.; Krause, H. Chem. Rev, 1994, 94, 1829.

6. Ohashi, K.; Nishi, N.J. Chem. Ph:s, 1998, 109, 3971.

7. Meol-Ner, M. J. Ph:s. Chem, 1980, 84, 2724.

8. Saigusa, H.; Lim, E. C. J. Phys. Chemi 1994, 98, 13470.

9. Inokuchi, Y.; Ohashi, K.; Matsumoto, M.; Nishi, N. J. Phys. Chem. 1995, 99, 3416.

10. Tsuzuki, S.; Uchimaru, T.; Matsumura, K.; Mikami, M.; Tanabe, K. Chem. Phss. Lett, $2000,319,547$.

11. Gonzalez, C.; Lim, E. C. J. Phis. Chem. A 2000, 104, 2953.

12. Lee, N. K.; Park, S.; Kim, S. K. J. Chem, Phss. 2002, 1/6, 7902.

13. Lee, N. K.; Park, S.; Kim, S. K. J. Chem. Phys. 2002, H6, 7910.

14. White, R. P.; Niesse, J. A.; Mayne, H. R. J. Chen. Phys. 1998. 108. 2208 .

15. Bouvier, B.; Brenner, V.; Millie, P.; Soudan, J.-M. J. Phys, Chem. A 2002, 106, 10326.

16. Piuzzi, F.; Dimicoli, I.; Mons, M.; Millie, P.; Brenner, V.; Zhao, Q.; Soep, B.; Tramer, A. Chent. Phss. 2002, 275, 123.

17. Song, J. K.; Lee, N. K.; Kim, S. K. Angew. Chem., Int. Ed. Engl. $2003,42,213$

18. Song, J. K.; Lee, N. K.; Kim, J. H.; Han, S. Y.; Kim, S. K. J. Chem. Phys, 2003, 119, 307।.

19. Mitsui, M.; Kokubo, S.; Ando, N.; Matsumolo, Y.; Nakajima, A.; Kaya, K. J. Chem. Phos. 2004, 121, 7553.

20. Frisch, M. J. et al. Gaussian 98; Gaussian, Inc.: Pittsburgh, PA, 1998.

21. Song. J. K.; Lee, N. K.; Han, S. Y.; Kim. S. K. J. Chem. Phss. $2002,117,9973$.

22. Song, J. K.; Han, S. Y.; Chu, I.; Kim, J. H.; Kim, S. K.; Lyapustina, S. A.; Xu, S.; Nilles, J. M.; Bowen, K. H. J. Chem. Phss. 2002, 116, 4777 .

23. Schiedt, J.; Weinkauf, R. Chem. Phys. Lett. 1997, 266, 201.

24. Shida. T.; Iwata. S. J. Chent. Phss, 1972, $56,2858$.

25. McHale, J.; Simons, J. J. Chem. Ph?s, 1980, 72, 425.

26. Kim, J. H.; Song J. K.; Park, H.; Lee, S. H.; Han, S. Y.; Kim, S. K. J. Chem. Phys. 2003, $119,4320$. 